\title{
Curvature-Induced Lateral Phase Segregation in Two-Component Vesicles
}

\author{
Udo Seifert \\ Department of Physics, Simon Fraser Unicersity, Burnaby, British Columbia, Canada V5A IS6 \\ and Institut für Festkörperforschung. Forschungszentrum Jülich, 5170 Jülich, Germany ${ }^{(\mathrm{a})}$ \\ (Received 2 November 1992)
}

\begin{abstract}
Shape transformations of two-component vesicles in which the spontaneous curvature depends on the local compositions in the two monolayers are investigated theoretically. Even if the two components do not exhibit lateral phase separation in spherical vesicles, temperature-induced shape transformations cause lateral phase segregation. Vesiculation of smaller buds is favored in the two-component system. In two-component vesicles with intermonolayer phase separation, thermal budding is dominated by the different thermal expansion coefficients of the two monolayers.
\end{abstract}

PACS numbers: $82.70 .-\mathrm{y}, 64.60 . \mathrm{Cn}, 68.15 .+\mathrm{e}$

Shape transformations of large single-component fluid bilayer vesicles have been investigated experimentally and theoretically (for reviews, see Refs. [1] and [2]). Even though not all aspects are completely understood so far, a comprehensive picture is emerging. The evolution of shapes is determined by the interplay between the bending energy $[3,4]$ of the bilayer membrane and the geometrical constraint of a controlled volume-to-area ratio of the vesicle. Changes in the latter quantity with temperature lead to pronounced shape transformations such as budding and vesiculation, depending on the area expansivity coefficients of the two monolayers, and their equilibrium area difference [5-11].

The next step towards an understanding of the physics of biological membranes, consisting of lipid mixtures, is the controlled study of two-component systems [12-20]. Quite generally, two different possibilities for a twocomponent membrane in the fluid state have to be distinguished: (i) The system can exhibit genuine phase separation, i.e., there are $A$-rich and $B$-rich domains separated by phase boundaries. The competition between the line tension of phase boundaries and the curvature energy can give rise to the formation of buds as recently discussed by Lipowsky [14]. (ii) There is no lateral phase separation. Even then, local deviations in the composition of the two monolayers lead to a spontaneous curvature if the two species have a different molecular geometry $[12,13,15,16]$. Suppose an initially spherical vesicle is subject to an increase in temperature. This temperature change necessarily leads to deviations from the spherical shape and, thus, to an inhomogeneous curvature. Because of the coupling between the local curvature and the local composition, the composition becomes inhomogeneous too. In this case, the shape change drives phase segregation contrary to case (i) where the domains cause the shape transformation. The purpose of this paper is to investigate case (ii) within a simple model which has the virtue of being exactly solvable since it can be mapped onto the standard model for single-component vesicles. So far, shape transformations of two-component vesicles have been studied explicitly only for two-dimensional vesicles $[15,17]$ and perturbatively in three dimensions around the sphere [18].

We first motivate the energy functional for a twocomponent vesicle. The composition (area fractions) of lipid $A$ in the individual monolayers $x_{A}^{i} \quad\left(=1-x_{B}^{i}\right)$ ( $i=$ in,out) can deviate locally from the mean value $\bar{x}_{A}$ as $\delta x_{A}^{i}(s) \equiv x_{A}^{i}(s)-\bar{x}_{A}$, where $s$ parametrizes the twodimensional surface of the vesicle. If this local deviation is different in the two monolayers, a local spontaneous curvature is induced according to $[15,16]$

$C_{0}(s)=\lambda\left(\delta x_{A}^{\text {out }}(s)-\delta x_{A}^{\text {in }}(s)\right)+\bar{C}_{0} \equiv \lambda \phi(s)+\bar{C}_{0}$,

with a coupling constant $\lambda$ with the dimensions of an inverse length. We also allow for a systematic spontaneous curvature $\bar{C}_{0}$.

The bending energy $F_{1}$ of the two-component vesicle is then chosen as a generalization of the bending energy of a single-component vesicle as

$$
\begin{aligned}
F_{1}=(\kappa / 2)\{ & \oint\left[2 H(s)-C_{0}(\phi(s))\right]^{2} d A \\
& \left.+\left[\alpha / 4 R^{2} D^{2}\right]\left(\Delta A-\Delta A_{0}\right)^{2}\right\},
\end{aligned}
$$

where $H(s)$ is the mean curvature and $\kappa$ is the bending rigidity which we assume for simplicity not to depend on the composition. For lateral homogeneous $C_{0},(2)$ is the area-difference-elasticity (ADE) model, within which budding and vesiculation have been investigated in detail $[7,11,21]$. It contains two bending contributions. The first term is the usual local bending energy [3]. The second term is the nonlocal bending energy which arises from the fact that while stresses in the monolayer can relax locally by gliding the monolayers over each other, a global term remains because of the closed topology [4]. It depends on the deviation of the area difference $\Delta A$ of the two monolayers from the equilibrium value $\Delta A_{0}$. While $\Delta A$ is a geometrical quantity depending only on the shape, $\triangle A_{0}$ is determined by the difference in the number of molecules of the outer and the inner mono- 
layer. $D$ is the distance of the two monolayers and the dimensionless constant $\alpha$ is of order unity.

Since the membrane does not show genuine phase separation, there is a free energy associated with deviation of the composition from its mean value. For small deviations, the lowest-order terms are $\sim\left[\delta x_{A}^{i 2}+\left(\xi \nabla \delta x_{A}^{i}\right)^{2}\right]$, where $\xi$ is the correlation length for composition fluctuations and $\boldsymbol{\nabla}$ is the covariant gradient operator. Expressed in the variable $\phi$, and ignoring the terms in $\left(\delta x_{A}^{\text {out }}\right.$ $\left.+\delta x_{A}^{\text {in }}\right)$, since they do not couple to the shape, the free energy $F_{2}$ associated with an inhomogeneous composition can be written as [16]

$$
F_{2}=(\kappa / 2) \epsilon \oint\left[\phi^{2}+(\xi \nabla \phi)^{2}\right] d A,
$$

where $\epsilon$ is some molecular energy divided by the bending rigidity. The total (free) energy $F$ is then $F \equiv F_{1}+F_{2}$. Since the $\phi$ field enters the energy $F$ quadratically, it can, in principle, be integrated out exactly. However, this involves an inversion of the $\nabla^{2}$ operator which is nontrivial for any nonspherical shape.

Axisymmetric vesicle shapes could now be calculated by first deriving the shape equations through variation of $F$ with respect to the shape and the $\phi$ field and then solving these equations in analogy to the single-component case $[8,21]$. Even though this approach does not pose any fundamental problems, a complete phase diagram would require extensive numerical work.

In this paper, we use another approach to determine solutions which minimize $F$. Since we are dealing with shape changes of large vesicles which have a linear size of the order of $\mu \mathrm{m}$ while the typical correlation length $\xi$ will be of the order of $\mathrm{nm}$, the gradient term in $F_{2}$ will be, in general, much smaller than the $\phi^{2}$ term. Therefore, we can set $\xi=0$. This allows us to map the energy $F$ onto the usual bending energy for single-component vesicles as follows: We minimize with respect to the composition profile $\phi(s)$. Since we assume that there is no exchange of molecules between the two monolayers, the constraint $\oint_{d A \phi}=0$ is added with a Lagrangian multiplier $\kappa \mu$ to $F$. Minimization then leads to $\phi(s)=[-\mu+\lambda(2 H(s)$ $\left.\left.-\bar{C}_{0}\right)\right] /\left(\lambda^{2}+\epsilon\right)$. Using the condition $\oint_{d A} A=0, \mu$ is found to be $\mu=\lambda\left(\triangle A /(A D)-\bar{C}_{0}\right)$, where $A \equiv 4 \pi R^{2}$ is the total area. This leads to

$$
\phi(s)=\left[2 \lambda /\left(\lambda^{2}+\epsilon\right)\right][H(s)-\Delta A / 2 D A],
$$

which shows that the local composition follows the deviation of the mean curvature $H(s)$ from its average value $\triangle A / 2 D A$. After inserting (4) into $F$, the total energy can be written as

$$
F=\frac{\kappa}{d \delta}\left\{\oint_{d A}(2 H)^{2}+\frac{\hat{\alpha}}{4 R^{2} D^{2}}\left(\Delta A-\widehat{\Delta A_{0}}\right)^{2}+\text { const }\right\} .
$$

Expression (5) is the standard form of the bending energy of a single-component vesicle in the ADE model [11] in which the three essential parameters have been renormalized as follows: First, the effective bending rigidity is $\kappa / \delta$ where

$$
\delta \equiv 1+\lambda^{2} / \epsilon>1
$$

measures the effective strength of the curvature to composition coupling. Thus, the bending rigidity decreases for a two-component system, as has been previously derived [16]. Second, the nonlocal term becomes more relevant for stronger couplings since $\alpha$ gets renormalized according to

$$
\hat{\alpha} \equiv \alpha+(\delta-1)(1+1 / \pi)>\alpha .
$$

Finally, the renormalized equilibrium area difference is

$$
\widehat{\Delta A_{0}} \equiv(\delta / \hat{\alpha})\left(4 \bar{C}_{0} D R^{2}+\alpha \Delta A_{0}\right) \text {. }
$$

This mapping of the energy $F$ of the two-component system onto the energy of a single-component system in the ADE model constitutes our main result. Since the ADE model has been investigated in detail, further analysis is relatively straightforward. As an illustrative example, consider the evolution of an initially spherical vesicle [with a homogeneous composition profile $\phi(s)=0$ ] with increasing temperature, see Fig. 1. For simplicity, we assume (i) that the thermal expansion coefficients of both monolayers are the same, (ii) that the membrane thickness $D$ decreases with temperature in such a way that the bilayer volume remains constant, and (iii) that the comparatively small thermal expansion of the enclosed fluid can be neglected [8]. Then the equilibrium area difference $\Delta A_{0}$ is related to the reduced volume $v$ by

$$
\Delta A_{0}(v)=\Delta A_{0}(1) / v,
$$

where $\Delta A_{0}(1)$ is the equilibrium area difference of the spherical vesicle $(v=1)$, as determined presumably by the (poorly understood) formation process of the vesicle. With increasing temperature the reduced volume decreases and the shape becomes more prolate. The inhomogeneous curvature then induces a nontrivial composition profile $\phi(s)$. In the outer monolayer, the $A$ molecules are enriched at the poles (if their enhancement in

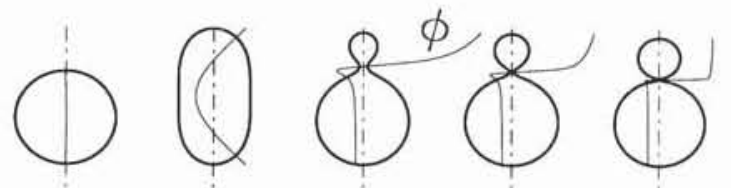

FIG. 1. Evolution of a spherical vesicle as the reduced volume $v$ changes due to an increase in temperature for $\bar{C}_{0}=0$, $\alpha=1, \hat{\alpha}=2$, and $\Delta A_{0}(1) / 8 \pi D R=1.7$. The thin curves show the composition $\phi$. The dash-dotted lines correspond to $\phi=0$. The reduced volumes are $v=1.0,0.89,0.89,0.86$, and 0.82 from left to right. At $v=0.89$, the symmetric and the asymmetric shapes have the same energy, indicating a discontinuous budding transition. The vesiculation line is reached with the last shape. 
the outer layer leads to a positive spontaneous curvature, i.e., if $\lambda>0$ ) while the $B$ lipids are enriched along the rim. For smaller $v$, the up/down asymmetric shapes have lower energy leading to a discontinuous budding transition. These shapes finally end up at the vesiculation point where two spheres of different radii are sitting on top of each other connected by a microscopic neck. In the vesiculated state, the composition within each sphere becomes homogeneous again with all the variation occurring in the neck [22]. Thus, the shape change, i.e., here budding and vesiculation, leads to phase segregation.

The extent to which the vesiculation, as an example of a shape transformation, is modified by the presence of two components is displayed in Fig. 2. It demonstrates that the formation of smaller buds is more favorable in the two-component system than in a one-component system under the same conditions. Specifically, we plot the (dimensionless) radius $r_{1}$ of the bud (where $r_{2}$ is the radius of the mother vesicle and $r_{1}^{2}+r_{2}^{2}=1$ ) at the vesiculation point as a function of the equilibrium area difference and the coupling constant $\delta$, where $\delta=1$ corresponds to the single-component case, i.e., no coupling. Analytically, the vesiculation point, $\widehat{\Delta A_{0}, L}\left(r_{1}\right)$, in the ADE model is given by [21]

$\widehat{\Delta A_{0, L}}\left(r_{1}\right)=2 D R\left[4 \pi\left(r_{1}+r_{2}\right)+2\left(1 / r_{1}+1 / r_{2}\right) / \hat{\alpha}\right]$.

Using Eqs. (7)-(10), it is easy to determine the relationship between $\Delta A_{0}(1), r_{1}$, and $\delta$ shown in Fig. 2. Note that vesiculation requires that the spherical vesicle has an initial equilibrium area difference $\Delta A_{0}(1)>8 \pi D R[1$ $+1 /(\pi \alpha)]$. For comparison, a sphere with no nonlocal bending energy has $\Delta A_{0}(1)=8 \pi D R$. Larger equilibrium area differences lead to smaller buds. For a given initial area difference, the radius $r_{1}$ of the bud decreases as the coupling increases.

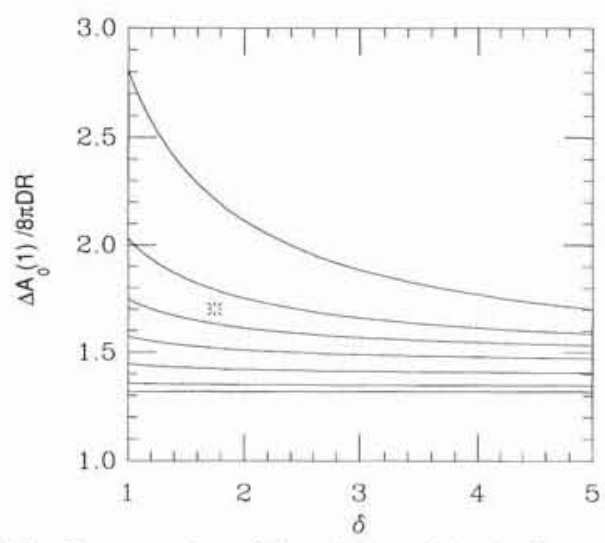

FIG. 2. Contour plot of the size $r_{1}$ of the bud at vesiculation as a function of the coupling $\delta$ and the initial equilibrium area difference $\Delta A_{0}(1)$. The contours correspond to $r_{1}=0.1,0.2$, $0.3,0.4,0.5,0.6$, and $\sqrt{2} / 2$ from top to bottom. Below the last curve, vesiculation is no longer possible. The asterisk marks the parameters chosen for the sequence shown in Fig. 1.
A crude estimate for the magnitude of the curvatureinduced phase segregation can be obtained as follows: If the spontaneous curvature is caused by the different molecular geometry of the $A$ and $B$ molecules, a typical value for the coupling $\lambda$ might be $\lambda=0.1 / \mathrm{nm}$. For the free energy density coefficient $\epsilon$, one estimates $\epsilon \simeq \tau T$ / $\kappa a^{2}$, where $\tau \equiv\left(T-T_{C}\right) / T_{C}$ is the distance to the $A-B$ critical point which separates the two cases alluded to in the introduction, and $a$ is a molecular length $a=1 \mathrm{~nm}$. With the typical value $T / \kappa \simeq 1 / 25$, one obtains $\delta-1$ $\simeq 0.25 / \tau$. For $\tau \lesssim 0.1$, this indicates an effect of $O(1)$ in $\delta$ which translates into an appreciable shift in $r_{1}$, see Fig. 2. More interesting, however, is to estimate the amount to which the phase segregation is effective. Inserting the values just given and $\tau \simeq 0.1$ into $\mathrm{Eq}$. (5), one obtains $\phi=14[H-\Delta A /(2 D A)] \mathrm{nm}$. With $H-\Delta A /(2 D A)$ $\simeq 1 / R$, the typical variation in the composition becomes of the order of $1 \%$ for vesicles with a radius $R \simeq 1 \mu \mathrm{m}$ but $10 \%$ for $R=100 \mathrm{~nm}$. The relatively small value for the larger vesicle can be substantially enhanced by approaching the critical point [23].

This model also covers the thermal evolution of an initially spherical vesicle formed spontaneously by intermonolayer phase separation in an $A$-rich outer monolayer and a $B$-rich inner monolayer as studied recently in experiment [19] and theory [20]. Again, for these vesicles, the spherical state has a lateral homogeneous composition in each monolayer. The different mean compositions $\bar{x}_{A}^{\text {in }} \neq \bar{x}_{A}^{\text {out }}$ cause a nonzero average spontaneous curvature $\bar{C}_{0}$. As temperature increases, the area excess leads to deviations from the spherical shape which induces again lateral phase segregation in the individual monolayers. Since for this case, the thermal expansion coefficients in the two monolayers are different due to their different composition, assumption (i) used above to derive the results shown in Fig. 2 no longer applies. In fact, it has been shown in Ref. [8] that any asymmetry $\gamma \gtrsim D / R$ in the thermal expansion coefficients of the two monolayers, $\alpha^{\text {in }}$ and $\alpha^{\text {out }} \equiv(1+\gamma) \alpha^{\text {in }}$, has a significant effect on the shape evolution for single-component vesicles. An analogous calculation for the case studied here shows that the size of the bud at vesiculation scales as

$$
r_{1} \approx\left\{\frac{D}{2 \delta \gamma \alpha \pi R /(2+\gamma)}\right\}^{1 / 3},
$$

for $D \ll R$ in units of $R$, if we start with an initially spherical relaxed vesicle $\left[\triangle A_{0}(1)=8 \pi D R\right]$. The temperature increase, $\Delta T$, necessary to reach vesiculation is $\Delta T$ $\approx r_{1}^{2} / \alpha^{\text {in }}(1+\gamma / 2)$. For an estimate, consider a $10 \%$ asymmetry in the expansion coefficients, i.e., $\gamma=0.1$, and choose $\alpha=1, \delta=2$. For large vesicles with $D / R=10^{-3}$, one obtains $r_{1} \simeq 0.1$, and, with $\alpha^{\text {in }} \simeq 5 \times 10^{-3} / \mathrm{K}, \Delta T \simeq 2^{\circ}$, i.e., an initially relaxed sphere reaches the vesiculation point after a $2^{\circ}$ temperature increase and the size of the bud is $\frac{1}{10}$ of the mother vesicle. The bud size and the temperature interval necessary to reach vesiculation are 
generically much smaller than for the case of symmetric expansion. Moreover, the asymmetric expansion also allows vesiculation even starting with a relaxed sphere $\Delta A_{0}(1)=8 \pi D R$ in contrast to the symmetric case as displayed in Fig. 2. However, the size of the vesicles obtained by spontaneous vesicle formation in mixed systems, so far, is typically $R \leqslant 100 \mathrm{~nm}$. In this case, $D / R \lesssim 10^{-2}$ and neither the bud radius $r_{1}$ nor the temperature interval $\Delta T$ will be substantially different from the case of symmetric expansion discussed above.

In summary, a model for vesicles made of two mixed components can be mapped onto the model for singlecomponent vesicles with renormalized coefficients. The formation of smaller buds is favored in the twocomponent system. Curvature-induced phase segregation should be a measurable effect for $R=100 \mathrm{~nm}$ vesicles or for larger vesicles in the vicinity of a critical point for demixing. For vesicles which show intermonolayer phase separation the same formalism applies. Thermal budding, however, is then dominated by the presence of different thermal expansion coefficients which, for large vesicles, typically lead to smaller buds and smaller temperature changes required to induce vesiculation.

Stimulating discussions with H.-G. Döbereiner, L. Miao, E. Sackmann, M. Wortis, and S. Langer who also gave the manuscript a critical reading are gratefully acknowledged. This work was funded by the National Science and Engineering Research Council of Canada.

(a) Permanent address.

[1] R. Lipowsky, Nature (London) 349, 475 (1991).

[2] M. Wortis, U. Seifert, K. Berndl, B. Fourcade, L. Miao, M. Rao, and R. K. P. Zia, in Proceedings of the Workshop on Dynamical Phenomena at Interfaces, Surfaces and Membranes, Les Houches, 1991, edited by D. Beysens, N. Boccara, and G. Forgacs (Nova Science, Commack, 1991)

[3] W. Helfrich, Z. Naturforsch. 28c, 693 (1973).

[4] E. Evans, Biophys. J. 14, 923 (1974).

[5] S. Svetina and B. Zeks, Eur. Biophys. J 17, 101 (1989).

[6] E. Evans and W. Rawicz, Phys. Rev. Lett. 64, 2094 (1990).

[7] W. Wiese and W. Helfrich, J. Phys. Condens. Matter 2, SA329 (1990); W. Wiese, W. Harbich, and W. Helfrich, J. Phys. Condens. Matter 4, 1647 (1992).
[8] K. Berndl, J. Käs, R. Lipowsky, E. Sackmann, and U. Seifert, Europhys. Lett. 13, 659 (1990); U. Seifert, K. Berndl, and R. Lipowsky, Phys. Rev. A 44, 1182 (1991).

[9] J. Käs and E. Sackmann, Biophys. J. 60, 825 (1991).

[10] L. Miao, B. Fourcade, M. Rao, M. Wortis, and R. K. P. Zia, Phys. Rev. A 43, 6843 (1991).

[11] U. Seifert, L. Miao, H.-G. Döbereiner, and M. Wortis, in The Structure and Conformation of Amphiphilic Membranes, edited by R. Lipowsky, D. Richter, and K. Kremer, Springer Proceedings in Physics Vol. 66 (Springer, Berlin, 1992), p. 93.

[12] S. H. Wu and H. M. McConnell, Biochemistry 14, 847 (1975).

[13] C. Gebhardt, H. Gruler, and E. Sackmann, Z. Naturforsch. 32c, 581 (1977).

[14] R. Lipowsky, J. Phys. II (France) 2, 1825 (1992).

[15] V. S. Markin, Biophys. J. 36, 1 (1981).

[16] S. Leibler, J. Phys. (Paris) 47, 507 (1986); S. Leibler and D. Andelman, J. Phys. (Paris) 48, 2013 (1987).

[17] D. Andelman, T. Kawakatsu, and K. Kawasaki, Europhys. Lett. 19, 57 (1992).

[18] T. Taniguchi, K. Kawasaki, D. Andelman, and T. Kawakatsu (to be published).

[19] E. W. Kaler, K. L. Herrington, A. K. Murthy, and J. A. N. Zasadzinski, J. Phys. Chem. 96, 6698 (1992).

[20] S. A. Safran, P. A. Pincus, D. Andelman, and F. C. MacKintosh, Phys. Rev. A 43, 1071 (1991); F. C. MacKintosh and S. A. Safran (to be published).

[21] L. Miao, U. Seifert, and M. Wortis (to be published).

[22] For the nearly vesiculated shape, where the composition gradient becomes large in the neck region, neglecting the $(\xi \nabla \phi)^{2}$ term in $F_{2}$ requires an additional justification. Indeed, even though $\nabla_{\phi}$ becomes large, the area element where this variation happens becomes small. In fact, connecting two spheres with a neck in the shape of the catenoid $(H=0)$ and solving for the optimal $\phi(s)$ on the catenoid in order to minimize the gradient term leads to a variational estimate for the energy stored in the neck. One finds $F_{1} \sim \rho$ and $F_{2} \sim 1 /|\ln \rho|$, where $\rho$ is the diameter of the neck. Thus, in the limit $\rho \rightarrow 0$ the total energy in the neck region vanishes.

[23] Very close to the critical point, the argument given above that the correlation length is small compared to the size of the vesicle is no longer valid. In fact, using $\xi \simeq a / \tau^{v}$, where $a \simeq 1 \mathrm{~nm}$ and $v=1$ is the critical exponent of the two-dimensional Ising model, one obtains $\tau_{\mathrm{c}} \approx a / R$ as a crossover temperature. For $\tau \lesssim \tau_{c}$, the gradient term in $F_{2}$ can no longer be neglected. 